\title{
SUR UNE CONJECTURE DE KRULL EN THEORIE DES VALUATIONS
}

\author{
P. RIBENBOIM*
}

Dans cette note, nous étudions les anneaux d'integrité primaires et complètement intégralement clos. Krull [1] a posé la question suivante: est-ce qu'un anneau d'integrité primaire et complètemente intégralement clos est nécéssairement un anneau de valuation?

Nagata [2] a répondu à cette question par la négative, en exhibant un exemple d'anneau d'integrité primaire, complètement intégralement clos, mais non de valuation. La démonstration que l'anneau de son exemple possédait effectivement les propriétés indiquées présentait néanmoins des lacunes non triviales à remplir. Ainsi, l'auteur a dedié une note [3] à l'élucidation de ces derniers points et à l'établissement de quelques propriétés plus précises satisfaites par cet anneau.

Malgré cette réponse négative à la conjecture de Krull, on pourrait néanmoins chercher des conditions supplémentaires pour qu'un anneau d'integrité primaire, complètement intégralement clos, soit un anneau de valuation. Ces conditions portent sur la structure de l'idéal premier unique de l'anneau et ont nature topologique.

Il est bien connu que si $A$ est un anneau d'integrité avec unité, ayant un seul idéal premier $\mathfrak{p}$ non trivial qui est principal, alors $A$ est l'anneau d'une valuation discrète. Nous remplaçons le fait que $\mathfrak{p}$ soit principal par la condition moins forte " $p$ est la réunion d'une suite croissante d'idéaux principaux" et determinons des conditions sur des telles suites pour assurer que $A$ soit un anneau de valuation. Ces conditions font intervenir la notion de puissance à exposant réel d'un idéal principal, qui avait été déjà considérée dans un cas distinct par Samuel [4].

La Proposition 1 n'est rien d'autre qu'un cas particulier d'un théorème récemment obtenu par Krull [5]. La Proposition 1 est employée pour la démonstration de la Proposition 2, qui dit: soit $A$ un anneau d'integrité primaire,

Received July 1, 1955.

* Boursier du Conselho Nacional de Pesquizas, Rio de Janeiro, Brasil. 
complètement intégralement clos, dont l'idéal premier $\mathfrak{p}$ est la réunion d'une suite croissante d'idéaux principaux $A t_{r}$; si tout idéal principal de $A$ est "asymptotiquement" une puissance (à exposant réel) des idéaux $A t_{r}$ (dans un sens qui sera précisé) alors $A$ est un anneau de valuation.

Quant à la Proposition 3, elle dit en gros: si, avec les hypothèses précedentes, la suite $\left(A t_{r}\right)$ est "bien choisie" de façon que

(C) la suite a une "largeur" nulle

(D) elle définit une topologie rendant continue la multiplication d'idéaux principaux,

alors $A$ est un anneau de valuation.

Naturellement, toutes les conditions indiquées sont aussi nécéssaires pour que $A$ soit un anneau de valuation.

Nous terminons l'article en faisant des commentaires qui montrent en particulier que les conditions introduites sont naturelles et d'aucune façon superabondantes.

\section{$\S 1$. Rappel des définitions et résultats}

Tous les anneaux considérés sont supposés des anneaux d'intégrité avec unité. Parmi les idéaux premiers, on exclut l'idéal nul et l'anneau lui-même.

Si $K$ est le corps des fractions de l'anneau $A$, un élément $x \in K$ est dit presque entier sur $A$ quand il possède la propriété suivante: il existe $a \in K$, non nul, tel que $a x^{n} \in A$ quelque soit $n$ entier positif. Un anneau $A$ est complètement intégralement clos quand il contient tous les éléments presque entiers sur $A$. Tout anneau complètement intégralement clos est intégralement clos, mais non réciproquement.

Un anneau (d'intégrité avec unité) $A$ est dit primaire quand il possède un seul idéal premier $\mathfrak{p}$; alors, le complémentaire de $\mathfrak{p}$ dans $A$ est l'ensemble des éléments inversibles (unités) de l'anneau $A$. En outre, tout idéal non trivial est un idéal primaire associé à $\mathfrak{p}$; en particulier, donnés $a, b \in \mathfrak{p}$, il existe un entier positif $n$ tel que $a^{n} \in A b$.

Toutes les valuations considérées sont supposées non triviales et de rang 1, c'est-à-dire, à valeurs réelles. Alors $A_{w}$ est l'anneau d'une valuation $w$ de $K$ si et seulement si $K$ est le corps des fractions de $A_{w}$, lequel est un sousanneau maximal de $K$, distinct de $K$. 
Pour la définition d'une valuation $w$ d'un corps $K$ il suffit de determiner une fonction $w_{0}$ à valeurs réelles, d'un sous-anneau $A$ de $K$ ayant corps des fractions $K$, et telle que :

$$
w_{0}(a b)=w_{0}(a)+w_{0}(b), \quad w_{0}(a+b) \geq \min \left\{w_{0}(a), w_{0}(b)\right\} \text { quand } a, b \in A .
$$

Si on pose, pour tout $x=\frac{a}{b} \in A$ (avec $\left.a, b \in A, b \neq 0\right) w\left(\frac{a}{b}\right)=w_{0}(a)-w_{0}(b)$, alors $w$ est une valuation de $K$, dont la restriction à $A$ est $w_{0}$.

Tout anneau de valuation (de rang 1) est complètement intégralement clos, néanmoins, il est faux que tout anneau complètement intégralement clos soit l'intersection d'anneaux de valuations [6]. Krull [1] a pourtant demontré, et nous en ferons usage, que si $A$ est primaire et complètement intégralement clos, alors il est intersection d'anneaux de valuations.

\section{§2. Notions préliminaires}

Soit $A$ un anneau primaire, $\mathfrak{p}$ son idéal premier, $K$ son corps des fractions.

Lemme 1. Si $t \in \mathfrak{p}$ alors $\bigcap_{n=1}^{\infty} A t^{n}=(0)$.

Notons $u=t^{-1}$; il suffira de montrer que $K=: \bigcup_{n=1}^{\infty} A u^{n}$, car alors $(0)=A: K$ $=A:\left(\bigcup_{n \geqq 1} A u^{n}\right)=\bigcap_{n \geqq 1}\left(A: A u^{n}\right)=\bigcup_{n \geqq 1} A t^{n}$.

Soit $B=\bigcup_{n \geqq 1} A u^{n}$; on a $A \subseteq B \leqq K, B$ est un anneau contenant $u=t^{-1}$; si $a \in A, a \neq 0$, et si $m$ est un entier tel que $t^{m} \in A a$ alors $a^{-1} \in A u^{m} \cong B$, donc $B=K$.

Remarquons que ce lemme est encore valable pour des idéaux $a=\left(a_{1}, \ldots\right.$, $\left.a_{m}\right)$ de type fini contenus dans $\mathfrak{p}$, car donné $t \in \mathfrak{p}$, non nul, il existe des entiers $q_{i}(i=1,2, \ldots, m)$, positifs, tels que $a_{i}^{q_{i}} \in A t$, donc il existe un entier $q>0$ tel que $\mathfrak{a}^{q} \subseteq A t$ et alors on a $\bigcap_{n \geqq 1} \mathfrak{a}^{n}=(0)$.

De la démonstration du lemme, il résulte:

Corollaire. Si $B$ est un sous-anneau de $K$, contenant $A$ et s'il existe un élément $t \in \mathfrak{p}$, non nul, tel que $t^{-1} \in B$ alors $B=K$.

Donnés les éléments $a \in K, a \neq 0$, et $t \in p, t \neq 0$, nous allons définir des nombres entiers et des nombres réels qui leur sont associés et qui joueront un important rôle dans cette note.

Soit $m_{t}(a)$ le plus grand des entiers $m$ (positifs ou négatifs) tels que 
$A a \cong A t^{m}$ (l'existence de $m_{t}(a)$ résulte du Lemme 1 , car $K=\bigcup_{m \geqq 1} A t^{-m}$ et (0) $\left.=\bigcap_{m \geqq 1} A t^{m}\right)$.

De même, soit $n_{t}(a)$ le plus petit des entiers $n$ (positifs ou négatifs) tels que $A t^{n} \leqq A a ;$ si $a \in A$, l'existence de $n_{t}(a)$ est triviale, si par contre $a=\frac{a_{1}}{a_{2}} \notin A$ (avec $a_{1}, a_{2} \in A$ ) alors $a_{1} \neq 0$ et si $n \geqslant 0$ est un entier tel que $t^{n} \in A a_{1}$ alors $a_{2} t^{n} \in A a_{1}$ donc $A t^{n} \cong A a$ et l'existence de $n_{t}(a)$ découle de $K=\bigcup_{n \geqq 1} A t^{-n}$.

On a $m_{t}(a) \leqq n_{t}(a)$; si $a \in A, a \notin \mathfrak{p}$, alors $m_{t}(a)=n_{t}(a)=0 ;$ si $a \in \mathfrak{p}$, alors $0 \leqq m_{t}(a), 0<n_{t}(a)$, et enfin $A a \subseteq A t$ équivaut à $0<m_{t}(a)$.

Supposons désormais que $A$ soit un anneau intégralement clos.

Soit $E_{t}(a)=\left\{\frac{m}{n} \mid A a^{n} \leqq A t^{m}\right\}, \quad F_{t}(a)=\left\{\frac{m}{n} \mid A t^{m} \leqq A a^{n}\right\}$ (nous conventionnons que les fractions $\frac{m}{n}$ sont toujours telles que $n>0$, le numérateur pouvant avoir signe quelconque).

Lemme 2. ( i ) Si $\frac{m}{n} \in E_{t}(a) \quad$ et $\frac{p}{q}<\frac{m}{n}$ alors $\frac{p}{q} \in E_{t}(a)$.

(ii) Si $\frac{m}{n} \in F_{t}(a) \quad$ et $\frac{m}{n}<\frac{p}{q}$ alors $\frac{p}{q} \in F_{t}(a)$.

(iii) Si $\frac{m}{n} \in E_{t}(a)$ et $\frac{p}{q} \in F_{t}(a)$ alors $\frac{m}{n} \leqq \frac{p}{q}$.

(i) Si $\frac{m}{n} \in E_{t}(a)$ et $\frac{p}{q}<\frac{m}{n}$ alors $A a^{n} \cong A t^{m}$ donc $A a^{q n} \cong A t^{q m}$ (car $q>0)$; or, de $p n<q m$ il résulte que $A t^{q m} \cong A t^{p n}$ donc $A a^{q n} \cong A t^{p n}$ et puisque $A$ est intégralement clos et $n>0$, on conclut que $A a^{q} \cong A t^{p}$, c'est-à-dire, $\frac{p}{q} \in E_{t}(a)$. (ii) se démontre analoguement. (iii) $\mathrm{Si} \frac{m}{n} \in E_{t}(a), \frac{p}{q} \in F_{t}(a)$, alors $A a^{n} \cong A t^{m}, A t^{p} \cong A a^{q}$; de $q>0$ et $n>0$ il vient $A a^{n q} \cong A t^{m q}, A t^{p n} \cong A a^{n q}$, c'est-à-dire, $A t^{p n} \leqq A t^{m q}$ d'où $p n \geqq m q$ et $\frac{m}{n} \leqq \frac{p}{q}$.

Si on note $\mu_{t}(a)=\sup E_{t}(a), \nu_{t}(a)=\inf F_{t}(a)$, alors $-\infty<\mu_{t}(a) \leqq \nu_{t}(a)$ $<+\infty, \operatorname{car} m_{t}(a) \in E_{t}(a), n_{t}(a) \in F_{t}(a)$. Si $a \in A, a \notin \mathfrak{p}$, on a $\mu_{t}(a)=\nu_{t}(a)$ $=0$; si $a \in \mathfrak{p}$ alors $0<\mu_{t}(a)$ (car si $q>0$ est un entier tel que $A a^{q} \subseteq A t$ alors $0<\frac{1}{q} \in E_{t}(a)$, donc $\left.0<\mu_{t}(a)\right)$. Remarquons qu'en général on peut avoir $\mu_{t}(a) \neq \nu t(a)$.

Lemme 3. Soit $s$ un nombre réel $a, a^{\prime} \in K, t \in \mathfrak{p}$, des éléments non nuls tels que $\mu_{t}(a)=\nu_{t}(a)=s$ et $\mu_{t}\left(a^{\prime}\right)=\nu_{t}\left(a^{\prime}\right)=s$. Alors $A a=A a^{\prime}$. 
En effet, soit $\Omega=(w)$ une famille de valuations de $K$ telles que $A=\bigcap_{w \in \Omega} A_{w}$ (car $A$ est intégralement clos). Si $\frac{m}{n} \in E_{t}(a), \frac{p}{n} \in F_{t}(a)$ (où $n>0$ ) alors $A t^{p} \cong A a^{n} \leqq A t^{m}$, donc $\frac{p}{n} w(t) \geqq w(a) \geqq \frac{m}{n} \cdot w(t) ;$ cela entraîne $\nu_{t}(a) \cdot w(t)$ $\geqslant w(a) \geqq \mu_{t}(a) \cdot w(t)$, donc $w(a)=s \cdot w(t)$ quelle que soit $w \in \Omega$. De même, $w\left(a^{\prime}\right)=s \cdot w(t)$, donc $w(a)=w\left(a^{\prime}\right)$ quelle que soit $w \in \Omega$, et alors $A a=A a^{\prime}$.

Corollaire. $A a=$ At équivaut à $\mu_{t}(a)=\nu t(a)=1$.

Le Lemme 3 permet de définir une opération de potentiation à exposants réels. Si $t \in \mathfrak{p}, t \neq 0$, on pose $A a=(A t)^{s}$ (où $s$ est un nombre réel de signe quelconque) quand on a $\mu_{t}(a)=\nu_{t}(a)=s$. Remarquons que cette opération n'est pas nécéssairement partout définie.

Lemme 4. Si $s_{1}, s_{2}, s$ sont des nombres réels strictement positifs, si $a, b \in \mathfrak{p}$, non nuls, alors:

( i ) si $s_{1} \leqq s_{2}$ et si $(A a)^{s_{1}},(A a)^{s_{2}}$ existent alors $(A a)^{s_{1}} \supseteqq(A a)^{s_{2}}$

(ii) $\quad s i(A a)^{s_{1}},(A a)^{s_{1} s_{2}}$ existent, alors $\left((A a)^{s_{1}}\right)^{s_{2}}$ existe et on a $\left((A a)^{s_{1}}\right)^{s_{2}}$ $=(A a)^{s_{1} s_{2}}$

(iii) si $(A a)^{s_{1}},(A a)^{s_{2}}$ existent, alors $(A a)^{s_{1}+s_{2}}$ existe et on a $(A a)^{s_{1}+s_{2}}$ $=(A a)^{s_{1}} \cdot(A a)^{s_{2}}$

(iv) si $(A a)^{s},(A b)^{s}$ existent, alors $(A a b)^{s}$ existe et on a $(A a b)^{s}$ $=(A a)^{s} \cdot(A b)^{s}$.

\section{Démonstration:}

(i) Si $(A a)^{s_{1}}=A b_{1},(A a)^{s_{2}}=A b_{2}$, alors le raisonnement du Lemme 3 entraîne $w\left(b_{1}\right)=w(a) s_{1}, w\left(b_{2}\right)=w(a) s_{2}$, donc $w\left(\frac{b_{2}}{b_{1}}\right)=w(a) \cdot\left(s_{2}-s_{1}\right) \geqq 0$ pour toute $w \in \Omega$; alors $\frac{b_{2}}{b_{1}} \in A$, c'est-à-dire $(A a)^{s_{2}} \leqq(A a)^{s_{1}}$.

(ii) Soit $(A a)^{s_{1}}=A b,(A a)^{s_{1} s_{2}}=A c$; alors $(A b)^{s_{2}}=A c$. En effet, du Lemme 3 on déduit que $w(b)=s_{1} \cdot w(a), w(c)=s_{1} s_{2} \cdot w(a)$, donc $w(c)=s_{2} \cdot w(b)$ quelle que soit $w \in \Omega$ et alors $(A b)^{s_{2}}=A c$.

(iii) Soit $(A a)^{s_{1}}=A b_{1},(A a)^{s_{2}}=A b_{2}$, donc $w\left(b_{1}\right)=w(a) \cdot s_{1}, w\left(b_{2}\right)=w(a) \cdot s_{2}$, et alors $w\left(b_{1} b_{2}\right)=w(a) \cdot\left(s_{1}+s_{2}\right)$ quelle que soit $w \in \Omega$, d'où $A b_{1} b_{2}=(A a)^{s_{1}+s_{2}}$.

(iv) Soit $(A a)^{s}=A a_{1},(A b)^{s}=A b_{1}$, donc $w\left(a_{1}\right)=w(a) \cdot s, w\left(b_{1}\right)=w(b) \cdot s$, et alors $w\left(a_{1} b_{1}\right)=w(a b) \cdot s$ quelle que soit $w \in \Omega$, c'est-à-dire, $(A a b)^{s}$ $=(A a)^{s} \cdot(A b)^{s}$. 


\section{§ 3. Résultats}

Soit $A$ un anneau primaire et complètement intégralement clos, p son idéal premier, $K$ son corps des fractions.

Puisque les résultats indiqués seront triviaux quand $\mathfrak{p}$ est un idéal principal, nous allons supposer implicitement que ce cas a été écarté. Remarquons que pour cela il suffit que $\mathfrak{p}$ possède une puissance contenue dans un idéal de type fini [5].

Nous cherchons à obtenir des conditions pour que $A$ soit un anneau de valuation. Nos conditions seront relatives à une suite croissante d'idéaux principaux $A t_{r}$ tels que $\mathfrak{p}=\bigcup_{r \geqq 1} A t_{r}$. Krull (loc. cit.) a envisagé des conditions analogues, mais portant sur l'ensemble filtrant des idéaux non nuls de type fini, contenus dans $\mathfrak{p}$.

Supposons donc qu'on ait $\mathfrak{p}=\bigcup_{r \geqq 1} A t_{r}$, où $\left(A t_{r}\right)$ est une suite strictement croissante d'idéaux principaux.

Pour tout élément $a \in K$, non nul, notons $m_{r}(a)=m_{t_{r}}(a), n_{r}(a)=n_{t_{r}}(a)$, etc. ... Posons $M(1)=1, M(r)=m_{2}\left(t_{1}\right) \cdot m_{3}\left(t_{2}\right) \ldots m_{r}\left(t_{r-1}\right) ;$ donc $M(r)$ $=M(r-1) \cdot m_{r}\left(t_{r-1}\right)$ et $M(r) \leqq m_{r}\left(t_{1}\right) \quad\left(\operatorname{car} A t_{1} \leqq A t_{2}^{m_{2}\left(t_{1}\right)}, A t_{2} \subseteq A t_{3}^{m_{3}\left(t_{2}\right)}, \ldots\right.$, $A t_{r-1} \subseteq A t_{r}^{m_{r}\left(t_{r-1}\right)}$, donc $A t_{1} \subseteq A t_{r}^{M(r)}$ et alors $\left.M(r) \leqq m_{r}\left(t_{1}\right)\right)$. De même, notons $N(1)=1, \quad N(r)=n_{2}\left(t_{1}\right) n_{3}\left(t_{2}\right) \ldots n_{r}\left(t_{r-1}\right) ;$ donc $N(r)=N(r-1) n_{r}\left(t_{r-1}\right)$ et $N(r) \geq n_{r}\left(t_{1}\right)$.

Remarquons que s'il existe un entier $N$ tel que $N \geqslant N(r)$ quelque soit $r \gtrsim 1$, alors de $A t_{r}^{N} \cong A t_{r}^{N(r)} \cong A t_{r}^{n_{r}\left(t_{r}\right)} \cong A t_{1}$ pour tout $r \geqq 1$ il résulte $\mathfrak{p}^{N} \cong A t_{1}$. Ainsi, pour écarter ce cas trivial, nous supposerons que $\lim _{r \rightarrow \infty} N(r)=\infty$.

Enfin, soit $\Omega=(w)$ une famille de valuations de $K$, telles que $A=\bigcap_{w \in \Omega} A_{w}$. Puisque $A$ est complètement intégralement clos, nous pouvons normaliser ces valuations de telle façon que $w\left(t_{1}\right)=1$ quelle que soit $w \in \Omega$.

Proposition 1. Soit A un anneau primaire et complètement intégralement clos. Supposons qu'il existe une suite strictement croissante d'idéaux principaux Atr tels que $\mathfrak{p}=\bigcup_{r \geqq 1} A t_{r}$ et satisfaisant à la condition suivante:

(A) pour tout $a \in \mathfrak{p}$, non nul, on $a \lim _{r \rightarrow \infty} \frac{m_{r}(a)}{n_{r}(a)}=1$.

Alors, $A$ est un anneau de valuation.

Démonstration:

Soit $a \in \mathfrak{p}$, non nul. De $A t_{r}^{n_{r}(a)} \cong A a \subseteq A t_{r}^{m_{r}(a)}, A t_{r}^{n_{r}\left(t_{1}\right)} \cong A t_{1} \subseteq A t_{r}^{m_{r}\left(t_{1}\right)}$, vient, 
en prenant $w \in \Omega$ arbitraire:

$$
\begin{aligned}
& n_{r}(a) \cdot w\left(t_{r}\right) \geqq w(a) \geqq m_{r}(a) \cdot w\left(t_{r}\right) \\
& n_{r}\left(t_{1}\right) \cdot w\left(t_{r}\right) \geqq 1=w\left(t_{1}\right) \geqq m_{r}\left(t_{1}\right) \cdot w\left(t_{r}\right) .
\end{aligned}
$$

Si on note $d_{r}(x)=\frac{n_{r}(x)-m_{r}(x)}{n_{r}(x)}$, où $x \in \mathfrak{p}$, non nul, alors $m_{r}(x)$ $=n_{r}(x)\left[1-d_{r}(x)\right]$. En faisant $x=a, x=t_{1}$, et en ermplaçant dans les inégalités (1), on obtient:

$$
\begin{aligned}
& n_{r}(a) \cdot w\left(t_{r}\right) \geqq w(a) \geqq w\left(t_{r}\right) \cdot n_{r}(a) \cdot\left[1-d_{r}(a)\right] \\
& n_{r}\left(t_{1}\right) \cdot w\left(t_{r}\right) \geqq 1=w\left(t_{1}\right) \geqq w\left(t_{r}\right) \cdot n_{r}\left(t_{1}\right) \cdot\left[1-d_{r}\left(t_{1}\right)\right] .
\end{aligned}
$$

Par division, il résulte que

$$
\begin{gathered}
\frac{n_{r}(a)}{n_{r}\left(t_{1}\right)} \cdot \frac{1}{1-d_{r}\left(\bar{t}_{1}\right)} \geqq w(a) \geqq \frac{n_{r}(a)}{n_{r}\left(t_{1}\right)} \cdot\left[1-d_{r}(a)\right] \\
w(a)=\lim _{r \rightarrow \infty} \frac{n_{r}(a)}{n_{r}\left(t_{1}\right)} .
\end{gathered}
$$

donc

Or, $w \in \Omega$ étant arbitraire et l'expression du deuxième membre étant indépendante de $w$, on aura, pour toute autre $w^{\prime} \in \Omega, w(a)=w^{\prime}(a)=\lim _{r \rightarrow \infty} \frac{n_{r}(a)}{n_{r}\left(t_{1}\right)}$. Or, ceci entraîne que $A$ est un anneau de valuation, car $A_{w}=A_{w}, \ldots \ldots=A$.

Proposition 2. Soit $A$ un anneau primaire et complètement intégralement clos. Supposons qu'il existe une suite strictement croissante d'ideaux principaux Atr tels que $\mathfrak{p}=\bigcup_{r \geqq 1} A t_{r}$ et satisfaisant la condition suivante:

(B) pour tout $a \in \mathfrak{p}$, non nul, on $a \lim _{r \rightarrow \infty} \frac{\mu_{r}(a)}{\nu_{r}(a)}=1$.

Alors, $A$ est un anneau de valuation.

\section{Démonstration:}

Il suffit de montrer que (B) entraîne (A). Soit $a \in \mathfrak{p}$, non nul. Soit $m^{\prime}$ l'entier tel que $m^{\prime} \leqq \mu_{r}(a)<m^{\prime}+1$ et de même, $n^{\prime}$ l'entier tel que $n^{\prime}-1<\nu_{r}(a)$ $\leqq n^{\prime}$. Alors, on a $A t_{r}^{m^{\prime}} \supseteqq A a \supseteqq A t_{r}^{n^{\prime}}$; de cela, il résulte que $m^{\prime} \leqq m_{r}(a)$ et $n_{r}(a) \leqq n^{\prime} ;$ enfin, de $m_{r}(a) \leqq \mu_{r}(a)$ et $\nu_{r}(a) \leqq n_{r}(a)$ il vient $m_{c}^{\prime}=m_{r}(a)$ et $n^{\prime}$ $=n_{r}(a)$.

Par l'hypothèse (B) on a $\lim _{r \rightarrow \infty}\left(1-\frac{\mu_{r}(a)}{\nu_{r}(a)}\right)=\lim _{r \rightarrow \infty} \frac{\nu_{r}(a)-\mu_{r}(a)}{\nu_{r}(a)}=0$. Or, $0 \leqq \frac{n_{r}(a)-m_{r}(a)}{n_{r}(a)} \leqq \frac{\left(\nu_{r}(a)+1\right)-\left(\mu_{r}(a)-1\right)}{\nu_{r}(a)}=\frac{\nu_{r}(a)-\mu_{r}(a)}{\nu_{r}(a)}+\frac{2}{\nu_{r}(a)}$, donc, si on a $\lim _{r \rightarrow \infty} \nu_{r}(a)=\infty$, il résulte que $\lim _{r \rightarrow \infty} \frac{n_{r}(a)-m_{r}(a)}{n_{r}(a)}=0$, c'est-à-dire, la con- 
dition (A) est vérifiée.

Montrons donc que $\lim _{r \rightarrow \infty} \nu_{r}(a)=\infty$. Or, d'abord, il est trivial que $F_{r}(a)$ $\supseteqq F_{r+1}(a)$, car si $0 \leqq \frac{m}{n} \in F_{r+1}(a)$ alors $A t_{r}^{m} \leqq A t_{r+1}^{m} \subseteq A a^{n}$, donc $\frac{m}{n} \in F_{r}(a)$. Il résulte que $\nu_{r}(a) \leqq \nu_{r+1}(a)$. S'il existait $N$ assez grand pour que $\nu_{r}(a) \leqq N$, pour tout $r \geqq 1$, alors $A t_{r}^{N} \cong A a$ pour tout $r \geqq 1$, donc $\mathfrak{p}^{N} \cong A a$, absurde !

Proposition 3. Soit A un anneau primaire et complètement intégralement clos. Supposons qu'il existe une suite strictement croissante d'idéaux principaux At $t_{r}$ tels que $p=\bigcup_{r \geqq 1} A t_{r}$ et satisfaisant les conditions suivantes:

(C) $\lim _{r \rightarrow \infty} \frac{M(r)}{N(r)}=1$.

(D) Si $a, b \in A, a, b \notin A t_{r+1}$, alors $a b \notin A t_{r}$ pour tout $r \geqslant 1$.

Alors $A$ est un anneau de valuation.

\section{Démonstration:}

Nous diviserons la démonstration en plusieurs parties.

(a) Pour tout $a \in \mathfrak{p}$, non nul, et $r \geqslant 1$, on a

$$
\frac{m_{r}(a)}{M(r)} \leqq \frac{m_{r+1}(a)}{M(r+1)} \quad \text { et } \quad \frac{n_{r+1}(a)}{N(r+1)} \leqq \frac{n_{r}(a)}{N(r)}
$$

En effet, de $a \in A t_{r}^{m_{r}(a)}, \quad t_{r} \in A t_{r+1}^{m_{r+1}\left(t_{r}\right)}$, vient $m_{r}(a) \cdot m_{r+1}\left(t_{r}\right)$ $=m_{r}(a) \cdot \frac{M(r+1)}{M(r)} \leqq m_{r+1}(a)$. De même, de $t_{r}^{n_{r}(a)} \in A a, t_{r+1}^{n_{r+1}} \in A t_{r}$, vient $n_{r}(a) \cdot n_{r+1}\left(t_{r}\right)=n_{r}(a) \cdot \frac{N(r+1)}{N(r)} \geq n_{r+1}(a)$.

(b) Pour tout $a \in \mathfrak{p}$, non nul, $\lim _{r \rightarrow \infty} \frac{m_{r}(a)}{M(r)}$ est fini.

Car $\lim _{r \rightarrow \infty} \frac{m_{r}(a)}{M(r)} \leqq \lim _{r \rightarrow \infty} \frac{n_{r}(a)}{N(r)} \cdot \lim _{r \rightarrow \infty} \frac{N(r)}{M(r)}$ et, en vertu de (a) et de (C) on déduit ( $b$ ).

(c) Posons: $w(a)=\lim _{r \rightarrow \infty} \frac{m_{r}(a)}{M(r)}$ quand $a \in \mathfrak{p}, a \neq 0 ; w(a)=0$ quand $a \in A$, $a \notin \mathfrak{p} ; w(0)=\infty$. Alors, si $a \in A$ on a $a \in \mathfrak{p}$ si et seulement si $w(a)>0$.

Car si $a \in \mathfrak{p}$ il existe $r$ tel que $a \in A t_{r}$, donc $m_{r}(a) \geqslant 1$ et $w(a)>0$.

(d) Si $a, b \in A$ alors $w(a+b) \geqslant \min \{w(a), w(b)\}$.

Nous pouvons supposer $a, b$ non nuls, dans $\mathfrak{p}$, sinon la formule est triviale. On a $a \in A t_{r}^{m_{r}(a)}, b \in A t_{r}^{m_{r}(b)} ;$ si $m_{r}^{\prime}=\min \left\{\mathrm{m}_{r}(a), m_{r}(b)\right\}$ alors $a+b \in A t_{r}^{m^{\prime} r}$ donc $m_{r}^{\prime} \leqq m_{r}(a+b)$. Si on avait $w(a+b)<\min \{w(a), w(b)\}$ alors pour $r$ assez 
grand il serait $\quad w(a+b)<\frac{m_{r}(a)}{M(\boldsymbol{r})}, \quad w(a+b)<\frac{m_{r}(b)}{M(\boldsymbol{r})}, \quad$ donc $\quad \frac{m_{r}(a+b)}{M(\boldsymbol{r})}$ $\leqq w(a+b)<\frac{m_{r}^{\prime}}{M(r)}$, contradiction!

(e) Si $x \in K, b \in A$, sont des éléments non nuls tels que $x b \in A$, alors il existe la limite $\lim _{r \rightarrow \infty} \frac{m_{r}(x)}{M(r)}$ et on a $w(x b)=\lim _{r \rightarrow \infty} \frac{m_{r}(x)}{M(r)}+w(b)$. En particulier, si $a, b \in A$, alors on a $w(a b)=w(a)+m(b)$.

Par hypothèse, $x=\frac{a}{b}$, où $a \in A, a \neq 0$. On a $x \in A t_{r}^{m_{r}(x)}, x \notin A t_{r}^{m_{r}(x)+1}$, donc si $u_{r}=t_{r}^{-1}$ alors $x u_{r}^{m_{r}(x)} \in A, x u_{r}^{m_{r}(x)} \notin A t_{r}$. De même, $b u_{r}^{m_{r}(b)} \in A$, $b u_{r}^{m_{r}(b)} \notin A t_{r} . \quad$ En vertu de $(\mathrm{D})$, on a $b x u_{r}^{m_{r}(b)+m_{r}(x)} \notin A t_{r-1}$, donc $b x u_{r}^{m_{r}(b)+m_{r}(x)}$ $\notin A t_{r}^{n_{r}\left(t_{r-1}\right)}$ (car $\left.A t_{r}^{n_{r}\left(t_{r-1}\right)} \cong A t_{r-1}\right)$, c'est-à-dire, $b x \notin A t_{r}^{m_{r}(b)+m_{r}(x)+n_{r}\left(t_{r-1}\right)}$ et alors $m_{r}(b)+m_{r}(x) \leqq m_{r}(b x)<m_{r}(b)+m_{r}(x)+n_{r}\left(t_{r-1}\right)$, la première inégalité étant triviale; il résulte que $m_{r}(b x)-m_{r}(b)-n_{r}\left(t_{r-1}\right)<m_{r}(x) \leqq m_{r}(b x)-m_{r}(b)$ et puisque $b x=a \in A$, on déduit, après division par $M(r)$, de la partie (b) et du fait que $\lim _{r \rightarrow \infty} \frac{n_{r}\left(t_{r-1}\right)}{M(\boldsymbol{r})}=\lim _{r \rightarrow \infty} \frac{1}{N(\boldsymbol{r}-1)} \cdot \lim _{r \rightarrow \infty} \frac{N(\boldsymbol{r})}{M(\boldsymbol{r})}=0$ l'existence de $\lim _{r \rightarrow \infty} \frac{m_{r}(x)}{M(\boldsymbol{r})}$ et l'égalité $w(b x)=w(b)+\lim _{r \rightarrow \infty} \frac{m_{r}(x)}{M(r)}$.

(f) Si on pose, pour tout $x=\frac{a}{b} \in K \quad($ avec $a, b \in A, b \neq 0) \quad w(x)=w(a)-$ $w(b)$, alors cette fonction $w$ ainsi prolongée à $K$, définit une valuation de $K$, dont l'anneau $A_{w}$ contient $A$. On a $w\left(\frac{a}{b}\right)=\lim _{r \rightarrow \infty} \frac{m_{r}\left(\frac{a}{b}\right)}{M(r)}$. Enfin, l'idéal de la valuation $w$ est égal à $\mathfrak{p}$.

Il suffit de montrer que si $x \in A_{w}, x \notin A$, alors $w(x)=0$. De $x \notin A$, il résult que $m_{r}(x) \leqq 0$ pour tout $r \geqslant 1$ ( $\operatorname{sinon}$, on aurait un indice $r \geqslant 1$, avec $m_{r}(x)>0$ donc $\left.A x \leqq A t_{r}^{m_{r}(x)} \leqq A t_{r} \leqq A\right)$; donc $w(x)=\lim _{r \rightarrow \infty} \frac{m_{r}(x)}{M(r)} \leqq 0$ et de $w(x) \gtrsim 0$ il vient $w(x)=0$.

(g) $A_{w}=A$.

En effet, supposons que $x \in A_{w}, x \notin A$. Alors, il existe $w^{\prime} \in \Omega$ tel que $x \notin A_{w}$, donc $A_{w} \neq A_{w}$, et ces anneaux de valuation étant maximaux, car $A$ est complètement intégralement clos, il existe $y \in A_{w}, y \notin A_{w}$; donc, $w(y)<0$, $w\left(y^{-1}\right)>0$, et alors $y^{-1} \in p$ d'après (f). Or, en vertu du corollaire au Lemme 1 , on conclut que $A_{w^{\prime}}=K$, absurde!

Corollaire. Soit $A$ un anneau primaire et intégralement clos. Supposons qu'il existe une suite croissante d'idéaux principaux $A t_{r}$ tels que $\mathfrak{p}=\bigcup_{r \geqq 1} A t_{r}$, 
satisfaisant les conditions $(C),(D)$. Alors, il existe un anneau de valuation (réelle) contenant tout anneau de valuation (de rang arbitraire) qui contient $A$.

En effet, remarquons que dans la démonstration de la Proposition 3 l'hypothèse que $A$ soit complètement intégralement clos n'a intervenu qu'à la partie $(\mathrm{g})$, et partout ailleurs on a employé seulement l'hypothèse moins forte que $A$ soit intégralement clos.

Soit donc $w$ la valuation (réelle) définie aux parties (a) - (f) ayant idéal égal à p. Si $A \subseteq A_{w^{\prime}} \neq A_{w}$, où $w^{\prime}$ est de rang arbitraire, soit $y \in A_{w}, y \notin A_{w}$, donc $w(y)<0, w\left(y^{-1}\right)>0$ et alors $y^{-1} \in \mathfrak{p}$; d'après le corollaire au Lemme 1 , il vient $A_{w^{\prime}}=K$, absurde!

\section{§4. Commentaires}

Nous indiquerons l'interpretation topologique de la propriété (D).

$\mathrm{Si}$, pour tout $r \geqq 1, V_{r}$ désigne l'ensemble complémentaire de $A t_{r}$ dans $A$, alors la famille d'ensembles $\left\{a V_{r} \mid a \in A, a \neq 0, r \geqslant 1\right\}$ est un système fondamental de voisinages pour une topologie sur $A^{*}$ (ensemble des éléments non nuls de $A$ ). En effet, tous les axiomes sont facilement vérifiés, par example, si $a V_{r}$ est un voisinage de $a$ et si on prend $U=a V_{r+1}$, alors si $b \in U$ on a $b V_{r+1}$ $\subseteq a V_{r}$, en vertu de la condition (D). Cette condition entraîne aussi la continuité de l'opération de produit, car $\left(a V_{r+1}\right)\left(b V_{r+1}\right) \cong a b V_{r}$ pour tout $r \geqq 1$. Enfin, cette topologie n'est pas séparée, car $\bigcap_{r \geqq 1} V_{r}$ est égal à l'ensemble des unités de $A$; mais, par passage au quotient, on obtient une topologie séparée, compatible avec le monoïde multiplicatif des idéaux principaux entiers non nuls de $A$. D'après cette interpretation, on voit comment est naturelle l'introduction d'une telle condition.

Enfin, dans la Proposition 3, les conditions ne sont pas superabondantes. En effet, l'exemple indiqué par Nagata montre l'existence d'un anneau primaire, complètement intégralement clos, dont l'idéal premier est la réunion d'une suite croissante d'idéaux principaux satisfaisant $(C)$ et néanmoins cet anneau n'est pas un anneau de valuation.

La vérification détaillée de ce fait peut être trouvée à la note [3]. Néanmoins, pour être complets, nous repéterons ici la façon de choisir la suite croissante d'idéaux principaux avec les propriétés voulues. Pour la notation, le lecteur doit se rapporter à l'article de Nagata [2], ou de l'auteur [3]. 
Soit $t \in K$ tel que $w(t)=1$; pour tout $r \geqslant 1$ soit $t_{r}=t^{\frac{1}{2^{r-1}}} \in K$ donc $w\left(t_{r}\right)$ $=\frac{1}{2^{r-1}}$ (l'existence de $t_{r}$ découle du fait que $K$ est algébriquement clos) et $\lim _{r \rightarrow \infty} w\left(t_{r}\right)=0$. Alors, l'idéal premier unique $\mathfrak{p}$ de l'anneau $A$ est la réunion de la suite strictement croissante d'idéaux principaux $A t_{r}$. En effet, donné $p \in A$ on sait qu'il existe la borne inférieure de l'ensemble des nombres réels $w_{\lambda}(p)$, $w_{e}(p)$ (quand $\left.\alpha \leqq \lambda \leqq 2 \alpha, e \in K, \alpha<w(e)<2 \alpha\right)$ et que cette borne inférieure est strictement positive. Donc, pour $r$ assez grand, on a $p \in A t_{r}$. En outre, il est clair que $m_{r}\left(t_{r-1}\right)=n_{r}\left(t_{r-1}\right)=2$ pour tout $r>1$, donc $M(r)=N(r)$ et la condition $(C)$ est vérifiée. Puisque $A$ n'est pas un anneau de valuation, alors il est impossible d'après la Proposition 3 que la condition $(D)$ soit vérifiée, par la suite $\left(A t_{r}\right)$. Remarquons qu'il est possible de construir explicitement des éléments $p, p^{\prime} \in A, p, p^{\prime} \notin A t_{r}$, mais tels que $p p^{\prime} \in A t_{r-1}$ (voir [3]).

\section{BIBLIOGRAPHIE}

[1] Krull, "Beiträge zur Arithmetik kommutativer Integritätsbereiche", II. Math. Zeits., 41 (1936), 665-679.

[2] Nagata, "On Krull's Conjecture concerning Valuation Rings," Nagoya Math. J., 4 (1952), 29-33.

[3] Ribenboim, "Sur une Note de Nagata relative à un Problème de Krull”, A paraitre dans le Math. Zeits.

[4] Samuel, "Some Asymptotic Properties of Powers of Ideals," Annals of Math., 56 (1952), $11-21$.

[5] Krull, "Eine Bemerkung über Primärintegritätsbereiche," A paraître dans le Math. Annalen.

[6] Nakayama, "On Krull's Conjecture concerning Completely Integrally Closed Integrity Domains," I, II. Proc. Imp. Acad. Tokyo, 18 (1942), 185-187, 233-236.

Mathematisches Institut der Universität, Bonn Instituto de Matemática Pura e Aplicada, Rio de Janeiro (Brasil) 